\title{
Moraxella catarrhalis Promotes Stable Polymicrobial Biofilms With the Major Otopathogens
}

\author{
Kirsten L. Bair ${ }^{1}$ and Anthony A. Campagnari, ${ }^{1,2 *}$ \\ ${ }^{1}$ Department of Microbiology and Immunology, Jacobs School of Medicine and Biomedical Sciences, University at Buffalo, \\ State University of New York, Buffalo, NY, United States, ${ }^{2}$ The Witebsky Center for Microbial Pathogenesis and Immunology, \\ University at Buffalo, State University of New York, Buffalo, NY, United States
}

\section{OPEN ACCESS}

Edited by: Giovanna Batoni, University of Pisa, Italy

Reviewed by:

Kristian Riesbeck, Lund University, Sweden

Rosalia Cavaliere,

University of Technology Sydney,

Australia

${ }^{*}$ Correspondence: Anthony A. Campagnari aac@buffalo.edu

Specialty section:

This article was submitted to

Antimicrobials, Resistance

and Chemotherapy,

a section of the journal

Frontiers in Microbiology

Received: 24 September 2019 Accepted: 13 December 2019

Published: 15 January 2020

Citation:

Bair KL and Campagnari AA (2020) Moraxella catarrhalis Promotes Stable Polymicrobial Biofilms With the Major Otopathogens. Front. Microbiol. 10:3006. doi: 10.3389/fmicb.2019.03006
Otitis media $(\mathrm{OM})$ is a prevalent pediatric infection characterized by painful inflammation of the middle ear. The Gram-negative diplococcus Moraxella catarrhalis is a commensal of the nasopharynx and one of three leading causative agents of OM. The most recent work on this multifaceted disease indicates that biofilms and polymicrobial infections play a pivotal role in recurrent and chronic OM, which are difficult to eradicate using standard antibiotic protocols. Although there have been significant advances in OM research, the actual bacterial and viral interactions leading to pathogenesis remain largely uncharacterized. However, colonization and persistence in the nasopharynx is clearly an essential first step. In this study, we assessed the role $M$. catarrhalis plays in the co-colonization and persistence of the other major otopathogens, Streptococcus pneumoniae and non-typeable Haemophilus influenzae (NTHi). We characterized both monomicrobial and polymicrobial biofilms using an in vitro nasopharyngeal colonization model. Biofilm assays were designed to mimic the nasopharynx and bacterial persistence was quantified over time. NTHi showed a steady and significant decline in viability over 20-48 h when this organism was in a dual species biofilm with $S$. pneumoniae. However, when $M$. catarrhalis was present in the polymicrobial biofilm NTHi survived for $48 \mathrm{~h}$ at $10^{7} \mathrm{CFU}$ per $\mathrm{mL}$. In addition, an isogenic M. catarrhalis catalase-deficient mutant was also fully capable of protecting NTHi from the bactericidal activity of $S$. pneumoniae in a polymicrobial biofilm. Our results show that $M$. catarrhalis promotes a favorable environment for stable polymicrobial biofilms by enhancing the survival of NTHi in the presence of $S$. pneumoniae. These data suggest that colonization with $M$. catarrhalis promotes stable co-colonization with other otopathogens.

Keywords: otitis media, biofilm, polymicrobial, Moraxella catarrhalis, Streptococcus pneumoniae, non-typeable Haemophilus influenzae

\section{INTRODUCTION}

There are more than 700 million cases of acute otitis media (AOM) diagnosed globally each year, with $50 \%$ of affected children under 5 years of age (Monasta et al., 2012). Moraxella catarrhalis, non-typeable Haemophilus influenzae (NTHi) and Streptococcus pneumoniae cause approximately $95 \%$ of $\mathrm{AOM}$ cases creating an incredible economic burden on healthcare 
systems (Broides et al., 2009). In the United States, it is estimated that AOM is responsible for 4.3 billion dollars in health-related costs (Tong et al., 2018). In addition to being the most common reason for doctor's office visits among children, $\mathrm{AOM}$ is also currently the most common reason for antibiotic use in the pediatric population. Recent studies have shown antibiotic resistance and decreased sensitivity developing among the major otopathogens (Pichichero, 2000a; Zielnik-Jurkiewicz and Bielicka, 2015; Sillanpaa et al., 2016; Korona-Glowniak et al., 2018). Further, the polymicrobial biofilms associated with AOM are incredibly resistant and difficult to treat using classic antibiotic protocols (Pichichero, 2000b; Leibovitz et al., 2003; Libson et al., 2005; Asher et al., 2008; Leibovitz, 2008; KoronaGlowniak et al., 2018). This is a result of conferred $\beta$-lactamase protection, quiescent bacteria within biofilms, poor antibiotic penetration and persister cells. When taken in combination with the continued prevalence of AOM in the post-vaccine era, these challenges demand novel preventative and treatment strategies.

Because all of these otopathogens can colonize asymptomatically, the interactions that occur in the nasopharynx that prevent or promote co-colonization play an important role in the steps that eventually lead to pathogenesis. Thus, we focused our studies on a more thorough evaluation of the possible events that occur during nasopharyngeal colonization. Providing a better understanding of the bacterial interactions that occur between the three primary otopathogens could lead to novel strategies for the prevention and treatment of AOM (Armbruster and Swords, 2010; Murphy, 2015).

To date, some of the dual species interactions of otopathogens have been characterized in vitro or in vivo. However, the ability to compare the three species in a single system has been limited by animal models that are less than ideal for the strict human pathogen $M$. catarrhalis. We have extended studies on these otopathogens by employing an in vitro nasopharyngeal colonization model adapted from previous studies originally designed for S. pneumoniae (Marks et al., 2012; Chao et al., 2017; Reddinger et al., 2018). The model mimics the conditions of the human nasopharynx including considerations for nasopharyngeal temperature, nutrient availability, aeration, and epithelial attachment.

Using this modified in vitro nasopharyngeal colonization model we assessed co-colonization dynamics of each otopathogen in dual species. Further, we analyzed interactions of all three otopathogens in triple species biofilms which have not been previously studied. Our results indicate that $M$. catarrhalis is able to promote survival of NTHi even in the presence of $S$. pneumoniae in triple species biofilms like those that have been previously shown to colonize the human nasopharynx (Hoa et al., 2009; Casey et al., 2010; Palmu et al., 2019).

\section{MATERIALS AND METHODS}

\section{Bacterial Strains and Culture Methods}

Moraxella catarrhalis strain 7169 is a clinical middle ear isolate (Faden et al., 1997). Minimally passaged planktonic M. catarrhalis cultures were grown at $37^{\circ} \mathrm{C}, 180 \mathrm{RPM}$, aerobically in chemically defined pneumococcal growth media (CDM) as previously described (van de Rijn and Kessler, 1980). NTHi strain 86-028NP is a clinical isolate from a pediatric patient who underwent a tympanostomy for chronic otitis media (OM) (Kennedy et al., 2000). NTHi cultures were grow at $37^{\circ} \mathrm{C}, 180 \mathrm{RPM}$, aerobically in CDM. S. pneumoniae EF3030 is a serotype 19F OM isolate that was grown statically and anaerobically in $\mathrm{CDM}$ at $37^{\circ} \mathrm{C}$ (Andersson et al., 1983). The co-infection strains M. catarrhalis 11-01-125, S. pneumoniae 11-01-125 and NTHi 11-01-125 were isolated from the middle ear fluid of a pediatric patient during a polymicrobial infection and generously provided by Michael Pichichero, MD (Rochester, NY, United States). Each species was grown as outlined above. These isolates were maintained for long term storage at $-80^{\circ} \mathrm{C}$.

\section{Static Biofilms and Time Course Assay}

Stationary in vitro biofilms were grown in 24-well plates on a monolayer of NCI-H292 bronchial carcinoma cells (ATCC CCL1848) as previously described (van Schilfgaarde et al., 1995). Planktonic cultures were grown to an $\mathrm{OD}_{600}$ of $\sim 0.2\left(\sim 10^{7} \mathrm{CFU}\right.$ per $\mathrm{mL})$. M. catarrhalis and NTHi were used at this concentration whereas S. pneumoniae was diluted 1:100 prior to seeding $\left(\sim 10^{4} \mathrm{CFU}\right.$ per $\left.\mathrm{mL}\right)$. These inocula were used for the studies in Figures 1B,C, 2. For the data shown in Figure 3, all three otopathogens were used at a starting inoculum of $\sim 10^{4} \mathrm{CFU}$ per mL. Wells received $350 \mu \mathrm{L}$ of each culture during seeding of either monomicrobial, dual species or triple species biofilms and CDM was added to a final volume of $1050 \mu \mathrm{L}$. Biofilms were incubated statically at $34^{\circ} \mathrm{C}$ and $5 \% \mathrm{CO}_{2}$. Media changes were completed at 4,20 , and $28 \mathrm{~h}$ post-seeding by replacing all spent media with $1 \mathrm{~mL}$ fresh CDM. Biofilm formation was quantified at $0,4,20,24$, and $48 \mathrm{~h}$ by carefully removing the supernatant and any planktonic or loosely attached bacteria and resuspending the biofilm in PBS via physical disruption with a pipette tip. Dilution plating onto selective media was utilized for CFU enumeration of $M$. catarrhalis (Mueller-Hinton agar + vancomycin at $3 \mathrm{mg}$ per $\mathrm{mL}$ ), $S$. pneumoniae (Blood agar + gentamicin at $4 \mathrm{mg}$ per $\mathrm{mL}$ ), and NTHi (Chocolate agar + clarithromycin at $2 \mathrm{mg}$ per $\mathrm{mL}$ ) at each time point. Plates were incubated for $24 \mathrm{~h}$ at $37^{\circ} \mathrm{C}$ and $5 \% \mathrm{CO}_{2}$.

\section{Transwell Assay}

Dual species S. pneumoniae EF3030 and NTHi 86-028NP biofilms were grown in 24-well plates on a fixed H292 cell monolayer. NTHi 86-028NP and S. pneumoniae EF3030 planktonic cultures were grown to an $\mathrm{OD}_{600}$ of $\sim 0.2$ and diluted 1:100 for a seeding concentration of $\sim 10^{5} \mathrm{CFU}$ per $\mathrm{mL}$ and $350 \mu \mathrm{L}$ of the 1:100 culture dilution was used for seeding. A transwell insert was added to each well [0.4 $\mu \mathrm{m}$ PET cell-culture 24-well adapted insert (Corning Inc., Corning, NY, United States)]. Transwell inserts of the treated wells received $350 \mu \mathrm{L}$ of $M$. catarrhalis 7169 prepared for seeding as discussed previously, and control wells were seeded with $350 \mu \mathrm{L}$ CDM. Media changes were completed at 4, 20, and $28 \mathrm{~h}$ post-seeding by replacing the spent media in the well and in the transwell insert separately. At 24 and $48 \mathrm{~h}$ the spent media was removed and the inserts were placed into a sterile 24 well plate. The biofilms in the 

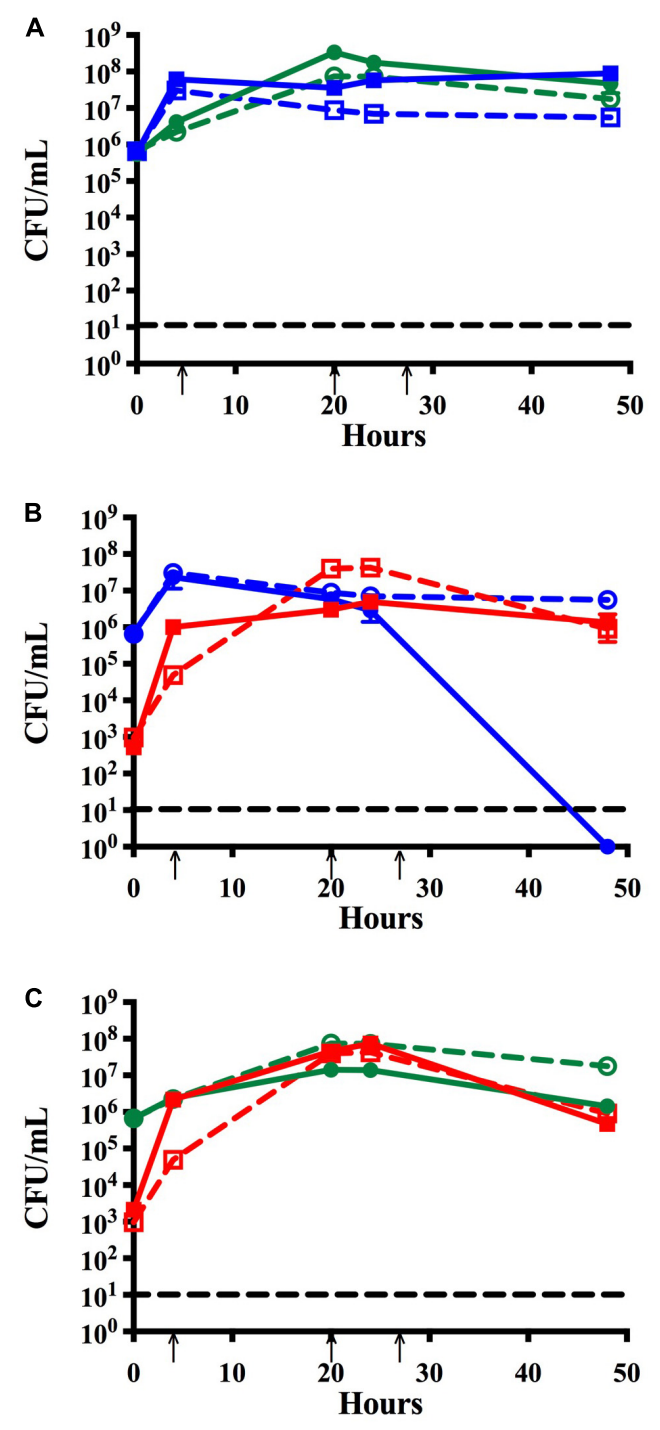

FIGURE 1 | Time course assay analyzing monomicrobial and dual species biofilms in vitro. Bacteria were grown in monomicrobial (dashed) or dual species (solid) biofilms and maintained with media changes (black arrows) for 48 h. (A) NTHi86-028 NP (blue) and M. catarrhalis 7169 (green) monomicrobial and dual species biofilms. (B) NTHi86-028 NP (blue) and S. pneumoniae EF3030 (red) monomicrobial and dual species biofilms. (C) M. catarrhalis 7169 (green) and S. pneumoniae EF3030 (red) monomicrobial and dual species biofilms. Black dashed line indicates the limit of CFU detection. Each symbol and error bar represent the mean CFU per $\mathrm{mL}$ and standard deviation (SD) of biofilms at the respective time point. Data presented are from three independent assays with a minimum of two biologic replicates per assay.

bottom of the 24-well plate and biofilms in the insert were each harvested in $1 \mathrm{~mL}$ PBS and dilution plated onto selective media for CFU enumeration as described above.

\section{Catalase Mutant Formation}

The $M$. catarrhalis catalase mutant $7169 \Delta \mathrm{katSpec1}$ was constructed in 7169 using the SOE-PCR technique to construct

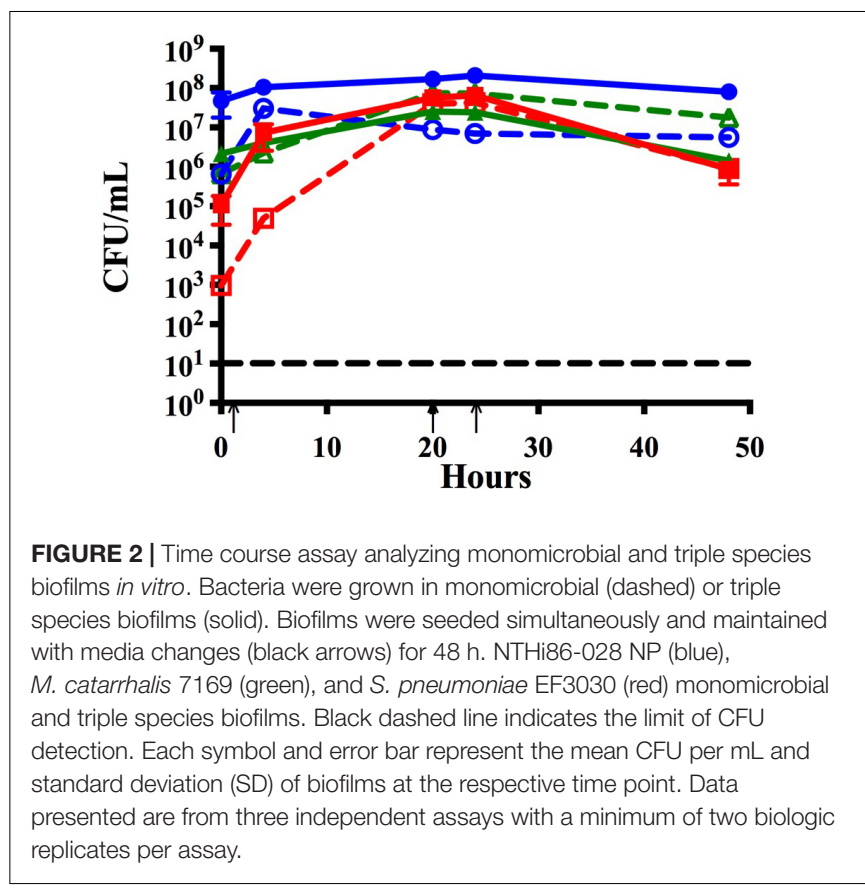

an insertion/deletion mutation (Thornton, 2016). In brief, amplicons containing 1350 nucleotides (nt) of genomic 7169 DNA upstream (using primers 2865/2868, Table 1) and $1364 \mathrm{nt}$ downstream (using primers 2869/2864, Table 1) of katA were amplified using the non-A-tailing high-fidelity polymerase PFU (Agilent Technologies, Santa Clara, CA, United States). A third $895 \mathrm{nt}$ amplicon was generated using primers 2870 and 2871 designed to flank the spectinomycin resistance cassette using pSpec as the template (Whitby et al., 1998; Plamondon et al., 2007). The $5^{\prime}$ region of primers 2868 and 2869 contained 20 nucleotides of homology to the primers 2870 and 2871 to allow for overlap and priming to give the final product containing the spectinomycin resistance cassette inserted within a $150 \mathrm{nt}$ deletion internal to the katA coding region. This was accomplished by a final PCR reaction using primers 2864 and 2865 and the three purified amplicons Qiagen MinElute Reaction Cleanup Kit (Qiagen, Germantown, MD, United States). The resulting 3609 nt amplicon, containing the insertionally inactivated katA, was purified Qiagen MinElute Reaction Cleanup Kit and used to mutagenize 7169 by natural transformation, as described (Furano and Campagnari, 2003). Transformants were selected following overnight growth on Mueller-Hinton agar plates containing $15 \mu \mathrm{g}$ per $\mathrm{ml}$ spectinomycin. Chromosomal DNA from transformant $7169 \Delta$ katSpec1 was isolated and subjected to PCR analysis (with primers 2874 and 2875 designed to flank the site of homologous recombination) and sequence analysis confirmed the integration of the inactivated katA construct into the 7169 genome. We further confirmed the functional inactivation using a qualitative catalase test. A sterile inoculating needle was used to transfer a single colony into a droplet of $3 \%$ hydrogen peroxide. A negative test is indicated by the lack of $\mathrm{O}_{2}$ production, which is a product of hydrogen peroxide catalysis by catalase. 

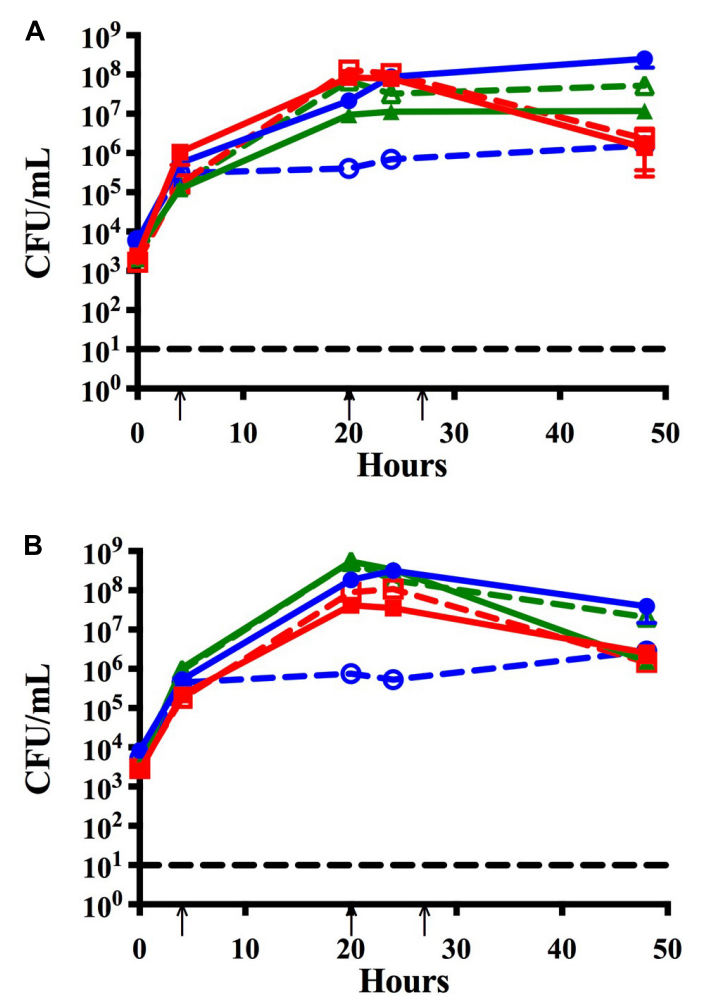

FIGURE 3 | Time course assay to assess the effects of lower seeding concentrations and address strain specificity by analyzing trends of co-infection isolates. Bacteria seeded simultaneously at a lower concentration $\left(\sim 10^{5} \mathrm{CFU}\right.$ per $\mathrm{mL}$ ) and maintained with media changes (black arrows) for $48 \mathrm{~h}$ in monomicrobial (dashed) or triple species (solid) biofilms. Each symbol and error bar represent the mean CFU per $\mathrm{mL}$ and standard deviation (SD) of biofilms at the respective time point. Data presented are from three independent assays with a minimum of two biologic replicates per assay. Black dashed line indicates the limit of CFU detection. (A) NTHi86-028 NP (blue), M. catarrhalis 7169 (green), and S. pneumoniae EF3030 (red) monomicrobial and triple species biofilms. (B) NTHi 11-01-125 (blue), M. catarrhalis 11-01-125 (green) and S. pneumoniae 11-01-125 (red) monomicrobial and triple species biofilms.

TABLE 1 | Nucleotide sequences of oligonucleotide primers used for PCR-based mutagenesis.

\begin{tabular}{ll}
\hline Primer & Sequence $\left(\mathbf{5}^{\prime} \mathbf{- \mathbf { 3 } ^ { \prime } )}\right.$ \\
\hline 2864 & ACCGATGCCGAAATGGTCTT \\
2865 & TIGCTGCACAGTTACCGC \\
2868 & CTCCTCACTATITGATAGACCTGATGAAACGCCTCTGG \\
2869 & GAAAACAATAAACCCTTGCCCGATGCCGAAGCTGAAATG \\
2870 & CCAGAGGCGTTCATCAGGTCTAATCAAAATAGTGAGGAG \\
2871 & CATTCAGCTTCGGCATCGGGCAAGGGTTATTGTITC \\
2874 & AAACTTCCCACATGCAACGC \\
2875 & CAACTAGAAGCACCGCCTCA
\end{tabular}

\section{Statistical Analyses}

Student's $t$-tests were used to compare the CFUs per mL of NTHi and S. pneumoniae exposed to $M$. catarrhalis or CDM in the filter well experiments. In addition, log transformed values of NTHi in the transwell assay (CDM treated, WT treated and catalase mutant treated) were checked for normal distribution using the Shapiro-Wilk test. Normally distributed data sets were analyzed by one-way ANOVA with Dunnett's multiple comparison test. Alternatively, data sets that did not pass the Shapiro-Wilk test were analyzed using the non-parametric Kruskal-Wallis with Dunn's multiple comparison test. All $P$-values were derived using a 95\% confidence interval. The statistical analysis was performed with Prism 8 from GraphPad Software, Inc. (La Jolla, CA, United States).

\section{RESULTS}

\section{Moraxella catarrhalis Promotes the Growth of NTHi in Dual Species Biofilms in vitro}

Previous studies have shown that colonization with $M$. catarrhalis occurs very early in life and $M$. catarrhalis is more likely to be present in polymicrobial AOM than infections resulting from a single bacterial species (Broides et al., 2009). Using our in vitro nasopharyngeal colonization model we assessed the relationship between $M$. catarrhalis and NTHi biofilms in physiologically relevant conditions. Biofilm formation was quantified over time via CFU enumeration. Figure 1A, shows that NTHi forms a slightly more robust biofilm, $\sim 1$ log greater, in dual species biofilms with $M$. catarrhalis than in monomicrobial biofilms which is consistent with previously reported data (Mokrzan et al., 2018). This is a statistically significant increase in viable NTHi at $48 \mathrm{~h}$ as compared to NTHi grown in monomicrobial biofilms (Supplementary Figure S1A).

\section{NTHi Is Unable to Persist in Biofilms With S. pneumoniae}

Although S. pneumoniae and NTHi each have a very high prevalence in OM individually, they are isolated together far less frequently (Casey et al., 2010). This is not surprising as previous studies have shown that peroxide production by planktonic S. pneumoniae elicits bactericidal effects on NTHi in vitro (Pericone et al., 2000). As recurrent OM is often considered a polymicrobial biofilm-associated disease, we performed studies to assess the relationship between these otopathogens in a dual species biofilm using our in vitro nasopharyngeal colonization model. Figure 1B shows that although NTHi can co-exist with $S$. pneumoniae for the initial $24 \mathrm{~h}$, there is a rapid decline in NTHi viability to undetectable levels by $48 \mathrm{~h}$. Based on these data, we concluded that NTHi and $S$. pneumoniae are unable to persist for extended periods in our in vitro model system.

\section{Moraxella catarrhalis and S. pneumoniae Form Dual Species Biofilms in vitro}

To determine if the $S$. pneumoniae bactericidal activity versus NTHi was also elicited against $M$. catarrhalis, we assessed the compatibility of these bacteria in biofilms using our in vitro 
model system. In contrast to the NTHi data, M. catarrhalis viability in dual species biofilms with $S$. pneumoniae remained consistent with the monomicrobial biofilm control (Figure 1C). At $48 \mathrm{~h}$ both species were present in the biofilm at $\sim 10^{6} \mathrm{CFU}$ per $\mathrm{mL}$ indicating that $M$. catarrhalis and $S$. pneumoniae can co-exist and persist in our model system in vitro. These data are consistent with previous in vivo studies suggesting that M. catarrhalis is capable of coping with the environmental stresses induced by $S$. pneumoniae in mixed species biofilms (Perez et al., 2014).

\section{Moraxella catarrhalis Promotes the Survival of NTHi in Polymicrobial Biofilms With S. pneumoniae}

As chronic or recurrent OM is considered a biofilm-associated disease, we further assessed the ability of these three otopathogens to co-exist and persist in a polymicrobial biofilm. Figure 2 demonstrates that NTHi is now fully capable of surviving and persisting in this triple species biofilm even in the presence of $S$. pneumoniae. The survival of NTHi at $48 \mathrm{~h}$ was stastically significant as compared to NTHi in dual species with S. pneumoniae (Supplementary Figure S1). It appears that the presence of $M$. catarrhalis in these polymicrobial biofilms provides some form of protection from the bactericidal effects of S. pneumoniae. Further, at $48 \mathrm{~h}$ NTHi forms the most robust biofilms of all three otopathogens as quantified by CFU per $\mathrm{mL}$.

Based on previous work done by Chao et al. (2017) we inoculated $S$. pneumoniae in all the previously mentioned time course assays at a lower seeding concentration to facilitate the analysis of bacterial-bacterial interactions. To ensure that our triple species data was not affected by lower seeding concentration, we completed a time course assay where all three otopathogens were diluted 1:100 and seeded simultaneously at approximately equivalent concentrations $\left(\sim 10^{4} \mathrm{CFU}\right.$ per $\left.\mathrm{mL}\right)$. Figure $3 \mathbf{A}$ confirms that the starting inocula had no effect on the establishment of polymicrobial biofilms and also confirms that the lower inoculum of $M$. catarrhalis was still able to protect NTHi from the bactericidal effects of the pneumococci.

\section{Multiple M. catarrhalis Clinical Isolates Protect NTHi in Polymicrobial Biofilms Containing S. pneumoniae}

We further assessed the strain specificity of the protective effect by testing co-infection strains that were simultaneously isolated from the middle ear fluid of a pediatric patient during a middle ear infection. Data collected from the co-infection strains demonstrated that NTHi was able to persist within the triple species biofilm and that protection by $M$. catarrhalis is not strain specific (Figure 3B).

\section{Cell to Cell Contact Is Not Required to Protect NTHi From the Bactericidal Effects of S. pneumoniae}

As one of the classic characteristics of bacterial biofilms is close contact at the cellular level, we utilized a transwell insert in our in vitro model system to physically isolate $M$. catarrhalis biofilms. Briefly, dual species S. pneumoniae and NTHi biofilms were seeded in 24-well plates on fixed H292 cell monolayers as described and transwell inserts were added to each well. $M$. catarrhalis was seeded into the inserts of the experimental wells and media alone was added to the control wells. Bacterial viability was assessed at 24 and $48 \mathrm{~h}$ as previously stated. Figure 4A shows that NTHi viability had a modest but significant decrease $(\sim 1 \log )$ at $24 \mathrm{~h}$ in the media treated controls as compared to the $M$. catarrhalis treated wells. This trend is even more apparent at $48 \mathrm{~h}$, which shows that NTHi viability in the control wells was decreased by $\sim 10^{3} \mathrm{CFU} / \mathrm{mL}$ compared with the $M$. catarrhalis exposed dual species biofilms which actually showed an increase in NTHi bacterial persistence to $\sim 10^{7} \mathrm{CFU}$ per $\mathrm{mL}$ (Figure 4B). These results demonstrate that $M$. catarrhalis can promote the survival and persistence of NTHi in the presence of $S$. pneumoniae and that this protective effect does not require direct contact. These data suggest that there is a factor(s) secreted or released into the supernatant that directly or indirectly confers protection to NTHi.

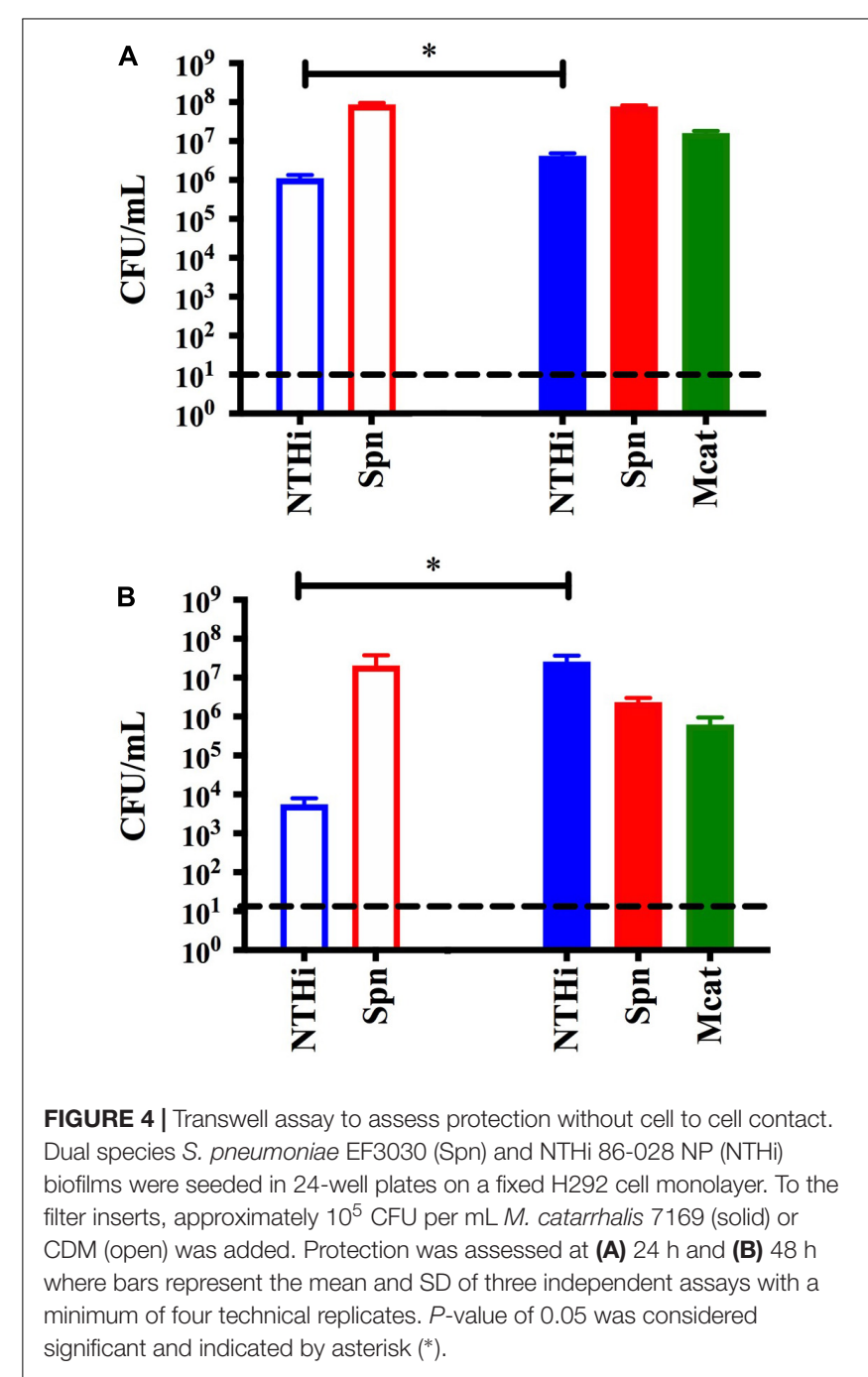




\section{Moraxella catarrhalis Catalase Production Is Not the Only Mechanism Involved in Protecting NTHi From Pneumococcal Hydrogen Peroxide}

Previous studies have shown that the bactericidal effects of $S$. pneumoniae hydrogen peroxide production on NTHi can be reversed by the addition of catalase (Pericone et al., 2000). Although NTHi produces catalase, the activity in vitro is considered quite low (Pericone et al., 2000) and this likely explains why it is not protective in our model system (Figure 1B). However, M. catarrhalis does produce a highly active catalase and this has been previously shown to neutralize the effects of $S$. pneumoniae hydrogen peroxide in planktonic cultures in vitro (Pericone et al., 2000). Based on this previous report, we constructed an isogenic catalase mutant in the $M$. catarrhalis 7169 (Mcat7169 $\Delta$ katASpec1) and confirmed this mutant was defective in catalase production (data not shown). This Mcat7169 $\Delta$ katASpec1 construct was assessed for protective activity in the transwell assay versus the wild-type Mcat7169 at $24 \mathrm{~h}$ (Figure 5A) and $48 \mathrm{~h}$ (Figure 5B). These results show that the Mcat7169 $\Delta$ katASpec1 mutant was able to confer protection of NTHi to the same extent as the wild type Mcat7169 at both time points. Given the previous reports, these results were somewhat surprising suggesting that catalase is not solely responsible for the protective effect seen in these polymicrobial biofilms using our model system.

\section{DISCUSSION}

The current model for OM pathogenesis is based on the concept that commensal bacteria colonize the nasopharynx of children very early in life and provide a reservoir for invasive diseases including AOM, sinusitis, and bacterial pneumonia. Although the mechanism of transition from colonization to opportunistic infection is not fully understood, it is widely accepted that changes in the host during a viral upper respiratory infection are linked to the subsequent onset of AOM (Heikkinen and Chonmaitree, 2003; Chonmaitree et al., 2008, 2016; Nokso-Koivisto et al., 2015). Based on this model, biofilms associated with colonization of otopathogens in the nasopharynx play a fundamental role in pathogenesis and are thus a primary target for preventative strategies.

While little is known about the transition from asymptomatic colonization to active infection, even less is known about how polymicrobial communities play a role in this transition. Using a model designed to mimic the environment of the nasopharynx we assessed the three primary otopathogens in dual species and triple species biofilms in vitro. To date, some of the dual species interactions have been characterized, but with contrasting results. For example, S. pneumoniae hydrogen peroxide production has been shown to have bactericidal effects on the other respiratory tract pathogens

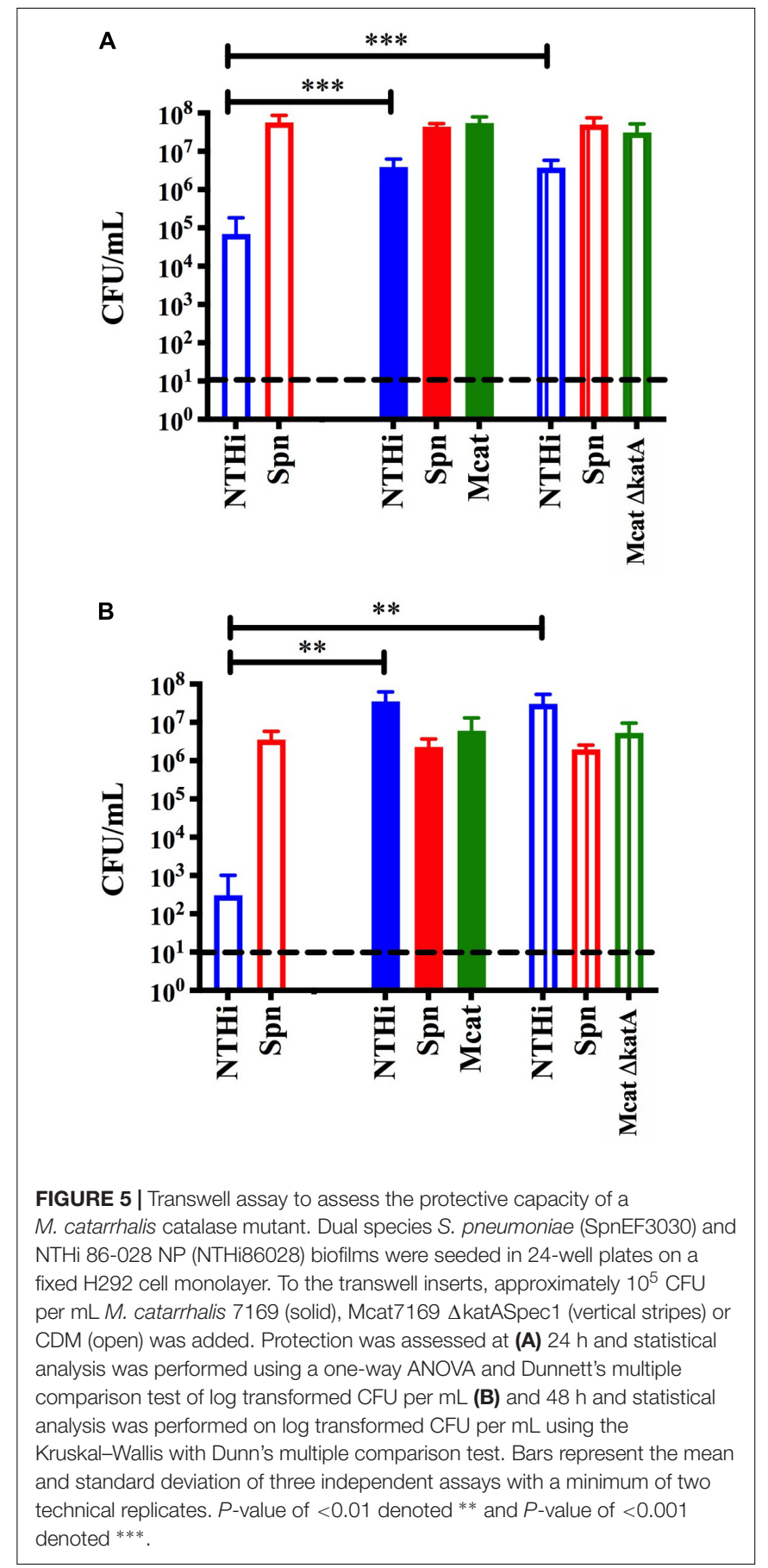

in planktonic cultures, in addition to being cytotoxic to the respiratory epithelial cells (Pericone et al., 2000). Likewise, $S$. pneumoniae produces neuraminidase which has the potential to remove sialic acid residues from other bacterial species exposing them to the host immune system. However, despite the many $S$. pneumoniae virulence factors cited, Perez et al. (2014) reported that S. pneumoniae increased colonization of $M$. catarrhalis in vivo. The same study also demonstrated that $M$. catarrhalis is able to confer 
$\beta$-lactamase protection to $S$. pneumoniae and conversely $S$. pneumoniae is able to provide $M$. catarrhalis with macrolide resistance. Based on the current literature, many questions remain relating to the potential synergism or antagonistic co-colonization of these two respiratory pathogens. The data presented in this study confirm that $M$. catarrhalis is in fact able to co-colonize in dual species biofilms with S. pneumoniae in vitro.

The multivalent pneumococcal conjugate vaccine and the Haemophilus influenzae type b vaccine have changed the landscape of nasopharyngeal colonization (Cohen et al., 2006; Revai et al., 2006; Dagan, 2009). Despite the success of these vaccines, NTHi strains and non-vaccine serotypes of S. pneumoniae remain a prominent cause of AOM. One of the few studies on the interactions of $S$. pneumoniae and NTHi suggests that NTHi can also confer $\beta$-lactamase-dependent antibiotic resistance and promote biofilm formation, and thus increase persistence, in vivo (Weimer et al., 2010, 2011). When studied in vitro, the interactions between S. pneumoniae and NTHi were reported to be cell density dependent and required the down regulation of pneumococcal genes regulating autolysis and fratricide (Hong et al., 2014). Our results indicate that $S$. pneumoniae is bactericidal to NTHi in dual species biofilms despite seeding at a relatively low concentration of S. pneumoniae to limit cytotoxicity. After approximately $24 \mathrm{~h}$ of biofilm growth, NTHi began to decline as a result of S. pneumoniae virulence.

We were also able to confirm that $M$. catarrhalis and NTHi successfully co-colonize in dual species biofilms as previously reported (Armbruster et al., 2010). Additionally, in our dual species model $M$. catarrhalis is able to promote the biofilm formation of NTHi supporting recently published work by Mokrzan et al. (2018). These observations have important implications because prolonged nasopharyngeal carriage subsequently increases the potential for transition to disease.

The nasopharynx is a polymicrobial environment. Multiple studies have shown that S. pneumoniae, M. catarrhalis and NTHi can simultaneously occupy this mucosal niche; however, very little is known about the bacterial interactions that occur between these species and the possible role these play in the pathogenesis of AOM (Hoa et al., 2009; Casey et al., 2010; Palmu et al., 2019). As mentioned previously, studies have shown that $M$. catarrhalis is more likely to be found in polymicrobial AOM infections than from a single-species infection (Broides et al., 2009). Additionally, colonization with $M$. catarrhalis happens very early in life, which is in part why $M$. catarrhalis is more frequently seen in children experiencing their first AOM episode as opposed to those with recurrent infections (Broides et al., 2009). These two qualities suggest that $M$. catarrhalis may be one of the first bacteria-bacteria interactions and a major factor in the subsequent colonization of other bacterial species. To test the possibility that $M$. catarrhalis may be important in polymicrobial colonization, we analyzed all three bacterial species in our colonization model in vitro. Our data confirmed that all three otopathogens were able to form a mixed species biofilm in our model system. More importantly, the presence of $M$. catarrhalis was essential for NTHi to survive the bactericidal effects of $S$. pneumoniae. This phenomenon, which appears to be "protective" for NTHi, has not been documented to date and provides novel insight into nasopharyngeal co-colonization and subsequently polymicrobial infection pathogenesis.

To explore the mechanism of protection we first considered the physical properties of the biofilms. We addressed the possibility that biofilm architecture was involved in shielding NTHi from S. pneumoniae or that cell to cell contact was required. We utilized a transwell assay which physically separated $M$. catarrhalis biofilms from NTHi and S. pneumoniae dual species biofilms, but allowed for the exchange of components in the supernatant. We found that physical contact is not necessary for $M$. catarrhalis to confer protection. These data suggest that a secreted or released factor(s) is responsible for the observed protective effect. We considered the known bactericidal effects of $S$. pneumoniae hydrogen peroxide production and hypothesized that catalase production may play a role in this process (Pericone et al., 2000). To test this, we constructed a catalase mutant in the $M$. catarrhalis 7169 background (Mcat7169 $\Delta$ katASpec1) and assessed its protective capacity in the transwell assay. The mutant had a phenotype similar to the wild-type demonstrating that catalase production by $M$. catarrhalis was not essential for protection. It is possible that Mcat7169 $\Delta$ katASpec1 was able to compensate using other mechanisms for coping with oxidative stress. Our initial results do not indicate a clear mechanism of protection, but they do suggest that it is reliant on a secreted or released factor(s) that is not catalase and is not dependent on physical or architectural characteristics of polymicrobial biofilms.

Future studies aim to use a more global approach to analyze the transcriptome of $M$. catarrhalis monomicrobial biofilms versus $M$. catarrhalis polymicrobial biofilms to parse out genes that are important in the polymicrobial environment and thus potentially important for the protective effect. The cumulative ability of these otopathogens to form polymicrobial biofilms, persist, and resist antibiotics has major implications in AOM epidemiology and pathogenesis. The mechanism of protection could provide a target for minimizing polymicrobial biofilms of the nasopharynx.

\section{DATA AVAILABILITY STATEMENT}

The raw data supporting the conclusions of this article will be made available by the authors, without undue reservation, to any qualified researcher.

\section{AUTHOR CONTRIBUTIONS}

$\mathrm{KB}$ performed all the aforementioned experimentation. $\mathrm{KB}$ and AC contributed to the conception of the experimental design and preparation of the manuscript. 


\section{FUNDING}

This work was supported by NIH R01DC014576 and NIH R01DC013554, awarded to AC.

\section{ACKNOWLEDGMENTS}

We would like to thank Lisa Hansen and Shauna Sauberan for technical assistance, Mary Canty and Chelsie Armbruster for guidance with statistical analyses, and Nicole Luke-Marshall for editorial insight.

\section{REFERENCES}

Andersson, B., Dahmen, J., Frejd, T., Leffler, H., Magnusson, G., Noori, G., et al. (1983). Identification of an active disaccharide unit of a glycoconjugate receptor for pneumococci attaching to human pharyngeal epithelial cells. J. Exp. Med. 158, 559-570. doi: 10.1084/jem.158.2.559

Armbruster, C. E., Hong, W., Pang, B., Weimer, K. E., Juneau, R. A., Turner, J., et al. (2010). Indirect pathogenicity of Haemophilus influenzae and Moraxella catarrhalis in polymicrobial otitis media occurs via interspecies quorum signaling. $m$ Bio 1:e0010210. doi: 10.1128/mBio.00102-10

Armbruster, C. E., and Swords, W. E. (2010). Interspecies bacterial communication as a target for therapy in otitis media. Expert Rev. Anti Infect Ther. 8, 1067-1070. doi: 10.1586/eri.10.109

Asher, E., Dagan, R., Greenberg, D., Givon-Lavi, N., Libson, S., Porat, N., et al. (2008). Persistence of pathogens despite clinical improvement in antibiotic-treated acute otitis media is associated with clinical and bacteriologic relapse. Pediatr. Infect. Dis. J. 27, 296-301. doi: 10.1097/INF.0b013e3181 5 ed79c

Broides, A., Dagan, R., Greenberg, D., Givon-Lavi, N., and Leibovitz, E. (2009). Acute otitis media caused by Moraxella catarrhalis: epidemiologic and clinical characteristics. Clin. Infect. Dis. 49, 1641-1647. doi: 10.1086/647933

Casey, J. R., Adlowitz, D. G., and Pichichero, M. E. (2010). New patterns in the otopathogens causing acute otitis media six to eight years after introduction of pneumococcal conjugate vaccine. Pediatr. Infect. Dis. J. 29, 304-309. doi: 10.1097/INF.0b013e3181c1bc48

Chao, Y., Bergenfelz, C., and Hakansson, A. P. (2017). In vitro and in vivo biofilm formation by pathogenic streptococci. Methods Mol. Biol. 1535, 285-299. doi: 10.1007/978-1-4939-6673-8_19

Chonmaitree, T., Revai, K., Grady, J. J., Clos, A., Patel, J. A., Nair, S., et al. (2008). Viral upper respiratory tract infection and otitis media complication in young children. Clin. Infect. Dis. 46, 815-823. doi: 10.1086/528685

Chonmaitree, T., Trujillo, R., Jennings, K., Alvarez-Fernandez, P., Patel, J. A., Loeffelholz, M. J., et al. (2016). Acute otitis media and other complications of viral respiratory infection. Pediatrics 137:e20153555. doi: 10.1542/peds.20153555

Cohen, R., Levy, C., de La Rocque, F., Gelbert, N., Wollner, A., Fritzell, B., et al. (2006). Impact of pneumococcal conjugate vaccine and of reduction of antibiotic use on nasopharyngeal carriage of nonsusceptible pneumococci in children with acute otitis media. Pediatr. Infect. Dis. J. 25, 1001-1007. doi: 10.1097/01.inf.0000243163.85163.a8

Dagan, R. (2009). Impact of pneumococcal conjugate vaccine on infections caused by antibiotic-resistant Streptococcus pneumoniae. Clin. Microbiol. Infect. 15(Suppl. 3), 16-20. doi: 10.1111/j.1469-0691.2009.02726.x

Faden, H., Duffy, L., Wasielewski, R., Wolf, J., Krystofik, D., and Tung, Y. (1997). Relationship between nasopharyngeal colonization and the development of otitis media in children. Tonawanda/Williamsville Pediatrics. J. Infect. Dis. 175, 1440-1445. doi: 10.1086/516477

Furano, K., and Campagnari, A. A. (2003). Inactivation of the Moraxella catarrhalis 7169 ferric uptake regulator increases susceptibility to the bactericidal activity of normal human sera. Infect. Immun. 71, 1843-1848. doi: 10.1128/iai.71.4.18431848.2003

\section{SUPPLEMENTARY MATERIAL}

The Supplementary Material for this article can be found online at: https://www.frontiersin.org/articles/10.3389/fmicb. 2019.03006/full\#supplementary-material

FIGURE S1 | A 48 h survival assessment. Bacterial species were statistically analyzed under each polymicrobial growth condition as compared to the monomicrobial control. Bars represent the mean and SD of minimally three independent assays. (A) NTHi, (B) S. pneumoniae, and (C) M. catarrhalis growth conditions were log transformed and analysis was completed using a student's t-test or a Mann-Whitney test based on the Shapiro-Wilk test for normality. $P$-value of $<0.05$ denoted $^{*},<0.01$ denoted $^{* *}$, and $<0.001$ denoted $* * *$.

Heikkinen, T., and Chonmaitree, T. (2003). Importance of respiratory viruses in acute otitis media. Clin. Microbiol. Rev. 16, 230-241. doi: 10.1128/cmr.16.2. 230-241.2003

Hoa, M., Tomovic, S., Nistico, L., Hall-Stoodley, L., Stoodley, P., Sachdeva, L., et al. (2009). Identification of adenoid biofilms with middle ear pathogens in otitisprone children utilizing SEM and FISH. Int. J. Pediatr. Otorhinolaryngol. 73, 1242-1248. doi: 10.1016/j.ijporl.2009.05.016

Hong, W., Khampang, P., Erbe, C., Kumar, S., Taylor, S. R., and Kerschner, J. E. (2014). Nontypeable Haemophilus influenzae inhibits autolysis and fratricide of Streptococcus pneumoniae in vitro. Microbes Infect. 16, 203-213. doi: 10.1016/j. micinf.2013.11.006

Kennedy, B. J., Novotny, L. A., Jurcisek, J. A., Lobet, Y., and Bakaletz, L. O. (2000). Passive transfer of antiserum specific for immunogens derived from a nontypeable Haemophilus influenzae adhesin and lipoprotein D prevents otitis media after heterologous challenge. Infect. Immun. 68, 2756-2765. doi: 10.1128/iai.68.5.2756-2765.2000

Korona-Glowniak, I., Zychowski, P., Siwiec, R., Mazur, E., Niedzielska, G., and Malm, A. (2018). Resistant Streptococcus pneumoniae strains in children with acute otitis media- high risk of persistent colonization after treatment. BMC Infect. Dis. 18:478. doi: 10.1186/s12879-018-3398-9

Leibovitz, E. (2008). Complicated otitis media and its implications. Vaccine 26(Suppl. 7), G16-G19. doi: 10.1016/j.vaccine.2008.11.008

Leibovitz, E., Greenberg, D., Piglansky, L., Raiz, S., Porat, N., Press, J., et al. (2003). Recurrent acute otitis media occurring within one month from completion of antibiotic therapy: relationship to the original pathogen. Pediatr. Infect. Dis. J. 22, 209-216.

Libson, S., Dagan, R., Greenberg, D., Porat, N., Trepler, R., Leiberman, A., et al. (2005). Nasopharyngeal carriage of Streptococcus pneumoniae at the completion of successful antibiotic treatment of acute otitis media predisposes to early clinical recurrence. J. Infect. Dis. 191, 1869-1875. doi: 10.1086/42 9918

Marks, L. R., Parameswaran, G. I., and Hakansson, A. P. (2012). Pneumococcal interactions with epithelial cells are crucial for optimal biofilm formation and colonization in vitro and in vivo. Infect. Immun. 80, 2744-2760. doi: 10.1128/ IAI.00488- 12

Mokrzan, E. M., Novotny, L. A., Brockman, K. L., and Bakaletz, L. O. (2018). Antibodies against the majority subunit (PilA) of the Type IV pilus of nontypeable Haemophilus influenzae disperse Moraxella catarrhalis from a dual-species biofilm. mBio 9:e02423-18. doi: 10.1128/mBio.02423-18

Monasta, L., Ronfani, L., Marchetti, F., Montico, M., Vecchi Brumatti, L., Bavcar, A., et al. (2012). Burden of disease caused by otitis media: systematic review and global estimates. PLoS One 7:e36226. doi: 10.1371/journal.pone.0036226

Murphy, T. F. (2015). Vaccines for nontypeable Haemophilus influenzae: the future is now. Clin. Vaccine Immunol. 22, 459-466. doi: 10.1128/cvi.00089- 15

Nokso-Koivisto, J., Marom, T., and Chonmaitree, T. (2015). Importance of viruses in acute otitis media. Curr. Opin. Pediatr. 27, 110-115. doi: 10.1097/MOP. 0000000000000184

Palmu, A. A., Ware, R. S., Lambert, S. B., Sarna, M., Bialasiewicz, S., Seib, K. L., et al. (2019). Nasal swab bacteriology by PCR during the first 24-months of life: a prospective birth cohort study. Pediatr. Pulmonol. 54, 289-296. doi: 10.1002/ppul.24231 
Perez, A. C., Pang, B., King, L. B., Tan, L., Murrah, K. A., Reimche, J. L., et al. (2014). Residence of Streptococcus pneumoniae and Moraxella catarrhalis within polymicrobial biofilm promotes antibiotic resistance and bacterial persistence in vivo. Pathog. Dis. 70, 280-288. doi: 10.1111/2049-632X.12129

Pericone, C. D., Overweg, K., Hermans, P. W., and Weiser, J. N. (2000). Inhibitory and bactericidal effects of hydrogen peroxide production by Streptococcus pneumoniae on other inhabitants of the upper respiratory tract. Infect. Immun. 68, 3990-3997. doi: 10.1128/iai.68.7.3990-3997.2000

Pichichero, M. E. (2000a). Acute otitis media: part II. Treatment in an era of increasing antibiotic resistance. Am. Fam. Phys. 61, 2410-2416.

Pichichero, M. E. (2000b). Recurrent and persistent otitis media. Pediatr. Infect. Dis. J. 19, 911-916. doi: 10.1097/00006454-200009000-00034

Plamondon, P., Luke, N. R., and Campagnari, A. A. (2007). Identification of a novel two-partner secretion locus in Moraxella catarrhalis. Infect. Immun. 75, 2929-2936. doi: 10.1128/iai.00396-07

Reddinger, R. M., Luke-Marshall, N. R., Sauberan, S. L., Hakansson, A. P., and Campagnari, A. A. (2018). Streptococcus pneumoniae modulates staphylococcus aureus biofilm dispersion and the transition from colonization to invasive disease. mBio 9:e02089-17. doi: 10.1128/mBio.02089-17

Revai, K., McCormick, D. P., Patel, J., Grady, J. J., Saeed, K., and Chonmaitree, T. (2006). Effect of pneumococcal conjugate vaccine on nasopharyngeal bacterial colonization during acute otitis media. Pediatrics 117, 1823-1829. doi: 10.1542/ peds.2005-1983

Sillanpaa, S., Sipila, M., Hyoty, H., Rautiainen, M., and Laranne, J. (2016). Antibiotic resistance in pathogens causing acute otitis media in Finnish children. Int. J. Pediatr. Otorhinolaryngol. 85, 91-94. doi: 10.1016/j.ijporl.2016. 03.037

Thornton, J. A. (2016). Splicing by overlap extension PCR to obtain hybrid DNA products. Methods Mol. Biol. 1373, 43-49. doi: 10.1007/7651_2014_182

Tong, S., Amand, C., Kieffer, A., and Kyaw, M. H. (2018). Trends in healthcare utilization and costs associated with acute otitis media in the United States during 2008-2014. BMC Health Serv. Res. 18:318. doi: 10.1186/s12913-018$3139-1$ van de Rijn, I., and Kessler, R. E. (1980). Growth characteristics of group a streptococci in a new chemically defined medium. Infect. Immun. 27, 444-448. van Schilfgaarde, M., van Alphen, L., Eijk, P., Everts, V., and Dankert, J. (1995). Paracytosis of Haemophilus influenzae through cell layers of NCI-H292 lung epithelial cells. Infect. Immun. 63, 4729-4737.

Weimer, K. E., Armbruster, C. E., Juneau, R. A., Hong, W., Pang, B., and Swords, W. E. (2010). Coinfection with Haemophilus influenzae promotes pneumococcal biofilm formation during experimental otitis media and impedes the progression of pneumococcal disease. J. Infect. Dis. 202, 1068-1075. doi: $10.1086 / 656046$

Weimer, K. E., Juneau, R. A., Murrah, K. A., Pang, B., Armbruster, C. E., Richardson, S. H., et al. (2011). Divergent mechanisms for passive pneumococcal resistance to beta-lactam antibiotics in the presence of Haemophilus influenzae. J. Infect. Dis. 203, 549-555. doi: 10.1093/infdis/jiq087

Whitby, P. W., Morton, D. J., and Stull, T. L. (1998). Construction of antibiotic resistance cassettes with multiple paired restriction sites for insertional mutagenesis of Haemophilus influenzae. FEMS Microbiol. Lett. 158, 57-60. doi: 10.1016/s0378-1097(97)00500-4

Zielnik-Jurkiewicz, B., and Bielicka, A. (2015). Antibiotic resistance of Streptococcus pneumoniae in children with acute otitis media treatment failure. Int. J. Pediatr. Otorhinolaryngol. 79, 2129-2133. doi: 10.1016/j.ijporl.2015.09.030

Conflict of Interest: The authors declare that the research was conducted in the absence of any commercial or financial relationships that could be construed as a potential conflict of interest.

Copyright ( 2020 Bair and Campagnari. This is an open-access article distributed under the terms of the Creative Commons Attribution License (CC BY). The use, distribution or reproduction in other forums is permitted, provided the original author(s) and the copyright owner(s) are credited and that the original publication in this journal is cited, in accordance with accepted academic practice. No use, distribution or reproduction is permitted which does not comply with these terms. 